\title{
Track Seek Control for Hard Disk Dual-Stage Servo Systems
}

\author{
Masahito Kobayashi and Roberto Horowitz, Member, IEEE
}

\begin{abstract}
A new decoupled track-seeking controller for a dualstage servo system of a magnetic disk drive has been developed. The controller is applied to the dual-stage system with a push-pull piezoelectric (PZT) micro-actuator and enables high-speed onetrack seeking and short-span track seeking. A one-track seeking controller was designed in three steps: 1) a voice coil motor (VCM) feedforward controller was designed to prevent overshoot in response of VCM actuator; 2) a feedforward loop from the VCM reference signal to the PZT actuator was designed in order to decouple the dual-stage actuator system; 3 ) a feedforward controller for the PZT actuator was designed such that an output PES (position error signal) only follows a PES reference signal. In the design of the short-span seeking controller, the PZT actuator's stroke limit was considered. Simulations and experiments show that the proposed dual-stage track-seeking controller performs better than the conventional single VCM servo.
\end{abstract}

Index Terms-Decoupled seeking control, dual-stage actuator, magnetic disk drive, motion control.

\section{INTRODUCTION}

$\mathbf{T}$ HE MOST significant trend in magnetic disk drives is that track density and storage capacity are increasing rapidly while access time is being reduced. This trend has led to the need for improved performance of the head-positioning servo system in order to accurately maintain the selected head position along the center of the track (track-following mode) and to provide rapid movement of the head from one track to another selected track (track-seeking mode). To maintain a high level of performance at a reduced track pitch, the servo system must be able to compensate disturbances caused by disk vibration, spindle run-out, the resonance modes of components, and external sources. This can only be accomplished by increasing the servo bandwidth and reducing the mass (weight) of the actuator. However, any major reduction of mass will lower actuator stiffness. Moreover, the servo bandwidth is limited mainly by the actuator's mechanical resonance modes. Studies of high-bandwidth servo systems have been made to design robust control systems for these mechanical resonance modes, multi-sensing control systems [10] that use an accelerometer or strain gauge as the vibration sensor, and dual-stage control systems that have an added small fine actuator to the conventional coarse VCM (voice coil motor) actuator.

Manuscript received July 17, 2000.

M. Kobayashi is with the Mechanical Engineering Research Laboratory, Hitachi, Ltd., 502 Kandatsu, Tsuchiura, Ibaraki, 300-0013, Japan (e-mail: masahito@merl.hitachi.co.jp).

R. Horowitz is with the Department of Mechanical Engineering, University of California, Berkeley, CA 94720 USA (e-mail: horowitz@me.berkeley.edu).

Publisher Item Identifier S 0018-9464(01)02532-8.



Fig. 1. Magnetic disk drive with dual-stage actuator.

Fig. 1 shows a schematic drawing of a 3.5-inch magnetic disk drive with a prototype of a push-pull piezoelectric (PZT) actuator [2]. This fine actuator is located between the head suspension and the base plate, which is moved by the VCM. A slider is attached to the tip of the suspension. The fine actuator is composed of multilayer PZT films and has two active polarized portions in a single body. When a voltage is applied to the active portions, one extends and the other contracts. The advantage of this moving suspension-type actuator is that it does not require large modifications to the shape of the head suspension assembly [2], [3]. However, the servo bandwidth is limited to about $3 \mathrm{kHz}$ because of the suspension's primary resonance mode. Two other types of dual-stage actuator are possible. The second-type micro-actuator is located between the suspension and slider [4], [5]. The advantage of this type is co-location of the actuator and sensor, so a servo bandwidth of $5 \mathrm{kHz}$ or more is possible. The third-type micro actuator is placed between the slider and the head element to drive the head element [6]. In theory, a large servo bandwidth can be achieved if the micro-actuator is located closer to the head. In practice, however, other considerations such as shock, flying height, manufacturability, and cost have a significant impact on the actuator choice and placement. In this study, we chose the moving suspension-type actuator (Fig. 1) for fine actuation and it achieves low driving voltage.

The mechanism for positioning the dual-stage actuator can improve both track-following and track-seeking performance. The VCM is used as a first stage to generate large but coarse and slow movement, while the micro actuator is used as a secondary stage to provide fine and fast positioning. A number of 


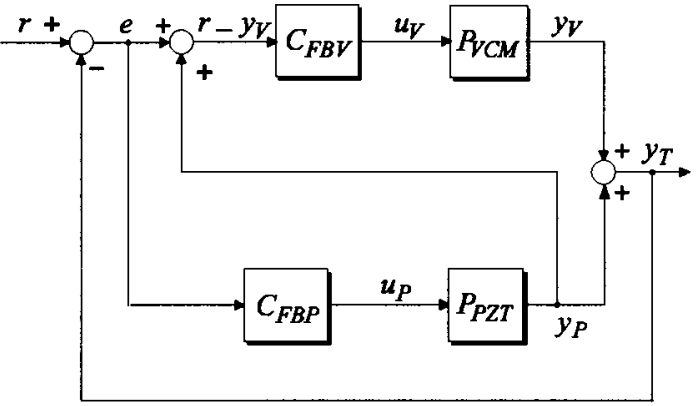

Fig. 2. Decoupled following controller.

control designs for track following have recently been discussed in several papers [1], [7]-[9]. However, track seeking performance of dual-stage servos has not been addressed much and it has only been evaluated based on step responses of a closed-loop track-following feedback system. This paper presents a new design for a track-seeking controller with a dual-stage actuator in magnetic disk drives. As the plant is a DISO (dual-input single-output) system, two separate reference signals are used for the VCM controller and the PZT controller and a decoupled seeking controller is used to drive each actuator independently by each reference signal during seeking operation. This paper focuses on one-track seeking and short-span track seeking operations. As the fine PZT actuator has a stroke limit of only a few micrometers, the seek controller design for the short-span seek operation should take into consideration the saturation properties of the PZT actuator.

Section II describes the structure of the dual-stage actuator system and the track-following controller design. Section III presents the proposed decoupled seeking controller design. Section IV discusses experimental results.

\section{Dual-Stage Track Following CONTROLler}

Several designs for track-following servos, which are a parallel type [7], [9], a master-slave type, a $\mu$-synthesis MIMO type [8], and a decoupled type [1], have been proposed. In this study, the decoupled track-following servo controller is used to achieve high-accuracy track following. Fig. 2 shows a block diagram of the servo system; $P_{\mathrm{VCM}}$ and $P_{\mathrm{PZT}}$ are, respectively, plant models of VCM and PZT actuators, and $C_{F B V}$ and $C_{F B P}$ are, respectively, controllers for the VCM and PZT actuators. The head position $y_{T}$ is a combination of the VCM output $y_{V}$ and the PZT output $y_{P}$. The output of the PZT actuator is added to the input of the VCM controller, which prevents the PZT actuator from going to the end of its stroke limit and maintains the output of the PZT on the center of the track.

The closed transfer function from a reference $r$ to the head position $y_{T}$ is given by

$$
y_{T}=\frac{P_{\mathrm{VCM}} C_{F B V}\left(1+P_{\mathrm{PZT}} C_{F B P}\right)+P_{\mathrm{PZT}} C_{F B P}}{\left(1+P_{\mathrm{VCM}} C_{F B V}\right)\left(1+P_{\mathrm{PZT}} C_{F B P}\right)} r
$$

The sensitivity function (error rejection function) $S_{T}$ is

$$
e=\frac{1}{\left(1+P_{\mathrm{VCM}} C_{F B V}\right)\left(1+P_{\mathrm{PZT}} C_{F B P}\right)} r .
$$

In the above equation, the total sensitivity function of the decoupled servo system is the product of the VCM and PZT
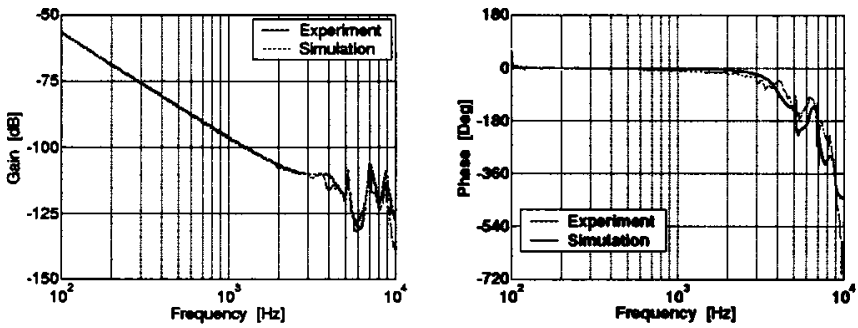

Fig. 3. Frequency response of VCM actuator.
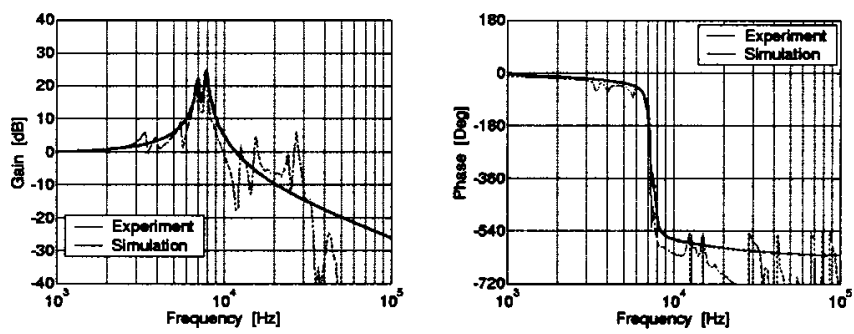

Fig. 4. Frequency response of PZT actuator.

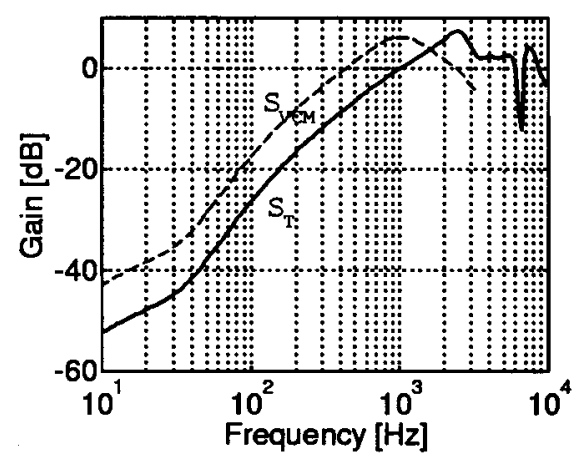

Fig. 5. Frequency response of sensitivity function.

loop sensitivities. Thus, the controller design can be decoupled into two independent controller designs, the VCM loop and PZT loop.

Usually, as the PZT actuator position $y_{P}$ is not detectable, the observer $\hat{P}_{\text {PZT }}$ is used to estimate the PZT actuator position from the input $u_{P}$ of the PZT actuator. The observer is designed to use the DC gain of the PZT actuator. In our multi-layer PZT actuator, the gain is $\pm 1.0 \mu \mathrm{m} / \pm 5 \mathrm{~V}$. The sampling period of the VCM actuator loop is $T_{s s}=154 \mu$ s and the PZT actuator loop is $T_{s}=38.5 \mu \mathrm{s}$ (four times faster).

We assumed that there is no mechanical coupling between the two actuators. Fig. 3 shows the experimental frequency responses of the VCM actuator (dotted line) and a simulated model $P_{\mathrm{VCM}}$ (solid line). The controller $C_{F B V}$ for VCM actuator is designed to use a phase-lag-lead compensator with analog notch filters and the open-loop crossover frequency is set at $400 \mathrm{~Hz}$, which has been changed from the usual $550 \mathrm{~Hz}$. Fig. 4 shows experimental frequency responses of the PZT actuator (dotted line) and simulated model $P_{\mathrm{PZT}}$ (solid line). The controller $C_{F B P}$ for the PZT actuator is designed to use a phase-lag compensator with two digital notch filters, which sets the crossover frequency at $1.5 \mathrm{kHz}$.

It can be seen in Fig. 5 that the sensitivity function $S_{T}$ has further attenuation of about $7 \mathrm{~dB}$ below $1 \mathrm{kHz}$, compared to the sensitivity function $S_{\mathrm{VCM}}$ of VCM loop. 


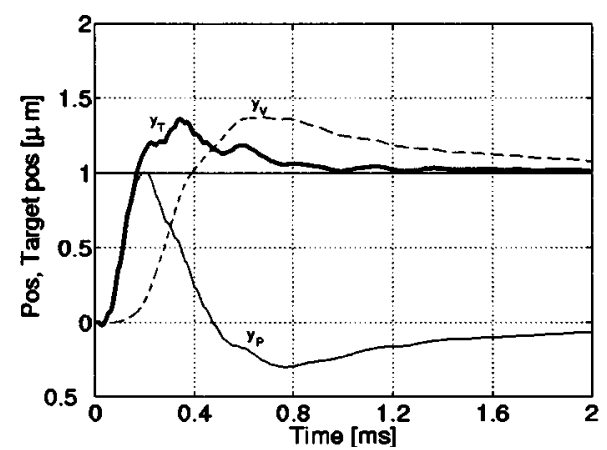

Fig. 6. 1- $\mu \mathrm{m}$ seeking response (preliminary step).

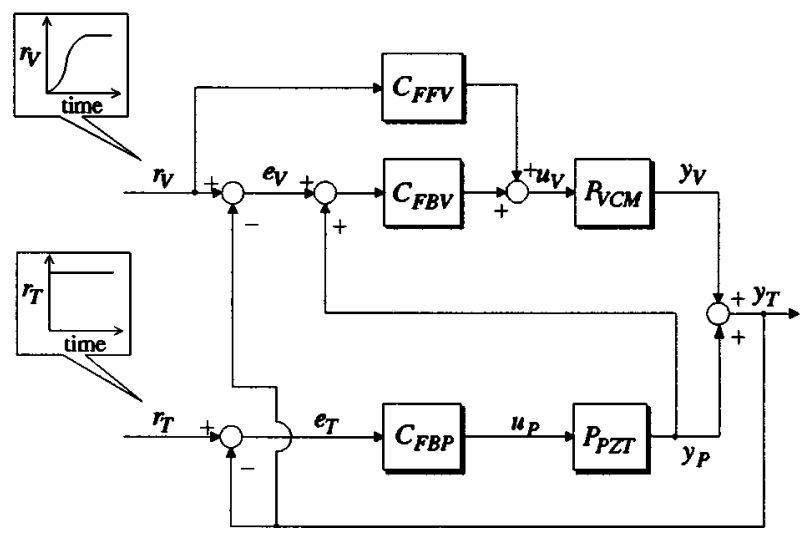

Fig. 7. Feedforward controller for VCM (Step 1).

\section{Dual-Stage Track-Seeking Controller}

High-speed one-track seeking and high-speed short-span seeking can improve data transaction performance. As the plant is a DISO (dual-input single-output) system, we use two separate reference signals for VCM position $y_{V}$ and the total head position $y_{T}$. We first explain one-track seeking design and then discuss the short-span seeking design.

\section{A. One-Track Seeking (Response Within PZT Stroke Limit)}

Our dual-stage actuator is the DISO system which has a VCM actuator input $y_{V}$, a PZT actuator input $y_{P}$, and a head position output $y_{T}$. The aim of the dual-stage seeking control system is to follow the head position $y_{T}$ in the specified reference trajectory $r_{T}$ by coordinating the movements of both the coarse VCM actuator and the fine PZT actuator. We used the same plant parameters and track-following controller as discussed in Section II, and assumed a density of $25.4 \mathrm{kTPI}$ (one-track pitch is $1 \mu \mathrm{m}$ ) for the simulation. For simplicity, we will explain in three steps design procedure.

Preliminary Step: The 1- $\mu \mathrm{m}$ step response of the decoupled track-following controller system (see Fig. 2) is shown in Fig. 6. Both the head position signal $y_{T}$ and the VCM actuator displacement $y_{V}$ are overshoot responses. The head position signal $y_{T}$ reaches the target in about $0.8 \mathrm{~ms}$, even though the head is moved by the high-response PZT actuator. The transfer function from $r$ to $y_{T}$ is given in (1).

Step 1: The servo system shown in Fig. 2 has only one reference trajectory $r$, which is fed into the closed loop system of both the VCM actuator and PZT actuator. As shown in Fig. 7,

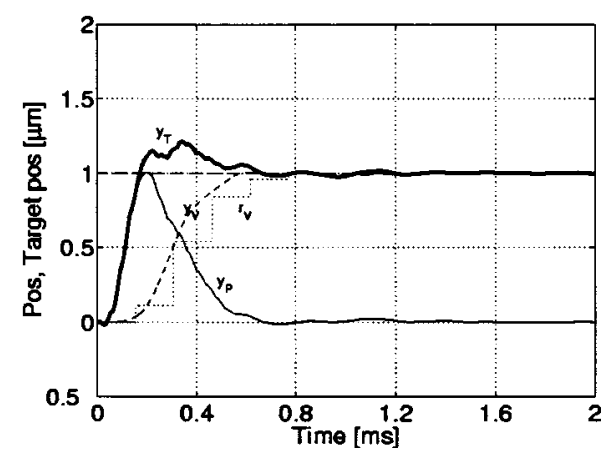

Fig. 8. 1- $\mu \mathrm{m}$ seeking response (Step 1).

two separate reference trajectories, $r_{V}$ for VCM actuator and $r_{T}$ for the head position, have been introduced. The transfer function from the two reference trajectories to the head position $y_{T}$ is

$$
\begin{aligned}
y_{T}= & \frac{P_{\mathrm{PZT}} C_{F B P}}{1+P_{\mathrm{PZT}} C_{F B P}} r_{T} \\
& +\frac{P_{\mathrm{VCM}} C_{F F V}+P_{\mathrm{VCM}} C_{F B V}}{\left(1+P_{\mathrm{VCM}} C_{F B V}\right)\left(1+P_{\mathrm{PZT}} C_{F B P}\right)} r_{V} .
\end{aligned}
$$

We designed feedforward controller $C_{F F V}$ with the VCM reference signal $r_{V}$ in order to prevent the overshoot of the VCM actuator response. The feedforward controller is designed by using the Zero Phase Error Feedforward Controller (ZPEFFC) [11]. Its target acceleration trajectory is a trapezoid-acceleration exponential-deceleration shape [11]. A step input feeds into the reference trajectory $r_{T}$ for head position. By using the ZFEFFC, we can establish that $C_{F F V} \approx P_{\mathrm{VCM}}^{-1}$, and

$$
\begin{aligned}
y_{V} & =\frac{P_{\mathrm{VCM}} \cdot C_{F F V}+P_{\mathrm{VCM}} \cdot C_{F B V}}{1+P_{\mathrm{VCM}} \cdot C_{F B V}} r_{V} \\
& \approx r_{V}
\end{aligned}
$$

can be given. The transfer function (3) from two target trajectories to the head position is then given by

$$
y_{T}=\frac{P_{\mathrm{PZT}} C_{F B P}}{1+P_{\mathrm{PZT}} C_{F B P}} r_{T}+\frac{1}{1+P_{\mathrm{PZT}} C_{F B P}} r_{V} .
$$

In Fig. 8, there is no overshoot of VCM response and it follows the discrete reference trajectory $r_{V}$, perfectly. The head position $y_{T}$, however, still exhibits overshoot behavior.

Step 2: Next, we designed feedforward pass compensator $C_{F F V P}$ from the reference signal $r_{V}$ to the PZT actuator input $u_{P}$ in order to decouple the DISO system (see Fig. 10). Let $C_{F F V P} \approx-\hat{P}_{\mathrm{PZT}}^{-1}$, where $\hat{P}_{\mathrm{PZT}}^{-1}$ is the inverse $\mathrm{DC}$ gain of the PZT actuator. The transfer function from reference signals to output $y_{T}$ can be described by

$$
\begin{aligned}
y_{T} & =\frac{P_{\mathrm{PZT}} C_{F B P}}{1+P_{\mathrm{PZT}} C_{F B P}} r_{T}+\frac{1+P_{\mathrm{PZT}} C_{\mathrm{FFVP}}}{1+P_{\mathrm{PZT}} C_{F B P}} r_{V} \\
& \approx \frac{P_{\mathrm{PZT}} C_{F B P}}{1+P_{\mathrm{PZT}} C_{F B P}} r_{T} .
\end{aligned}
$$

In (6), only the reference trajectory $r_{T}$ and the closed loop system of the PZT actuator influence the head position $y_{T}$. It is not affected by the VCM reference trajectory $r_{V}$. The output of the PZT actuator $y_{P}$ has offset response, because there is no 


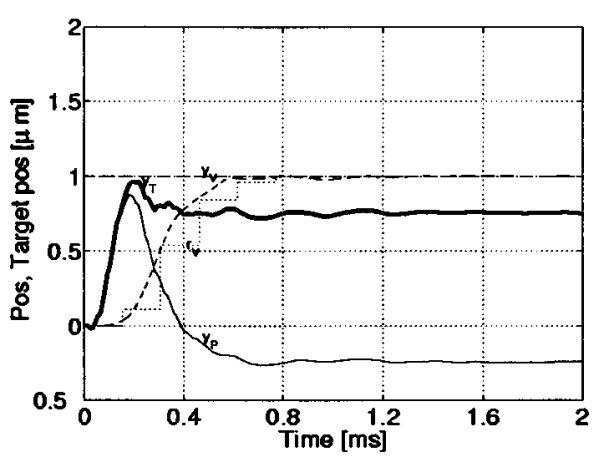

Fig. 9. 1- $\mu \mathrm{m}$ seeking response (Step 2).

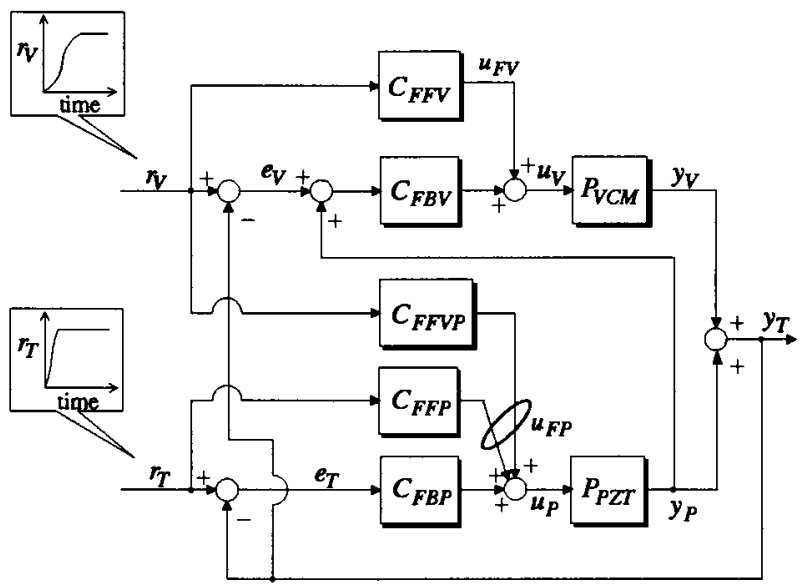

Fig. 10. Feedforward controller for PZT (Step 3).

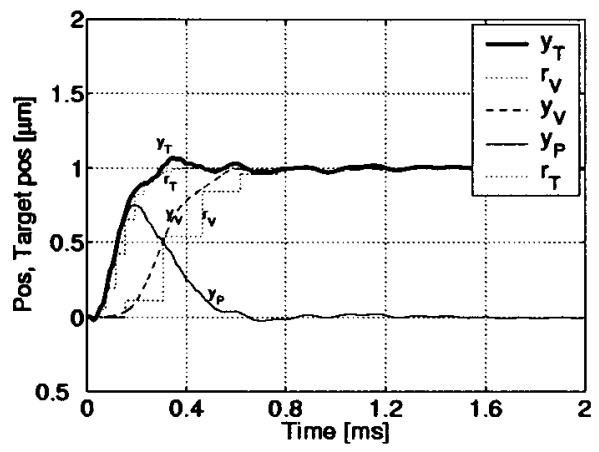

Fig. 11. 1- $\mu \mathrm{m}$ seeking response (Step 3).

high gain at low frequencies in the PZT loop system, as shown in Fig. 9.

Step 3: Finally, we designed feedforward compensator $C_{F F P}$ for the PZT actuator such that the output $y_{T}$ only follows the reference trajectory $r_{T}$, as shown in Fig. 10. If we suppose $C_{F F P} \approx \hat{P}_{\mathrm{PZT}}^{-1}$, the transfer function from reference signals to output $y_{T}$ is

$$
\begin{aligned}
y_{T} & =\frac{P_{\mathrm{PZT}} C_{F F P}+P_{\mathrm{PZT}} C_{F B P}}{1+P_{\mathrm{PZT}} C_{F B P}} r_{T} \\
& \approx r_{T} .
\end{aligned}
$$

The output head position $y_{T}$ is only controlled by one reference trajectory $r_{T}$. Fig. 11 indicates that the proposed decoupled seeking controller greatly improves the responses. It takes about $0.3 \mathrm{~ms}$ to move the head to the desired track so that the

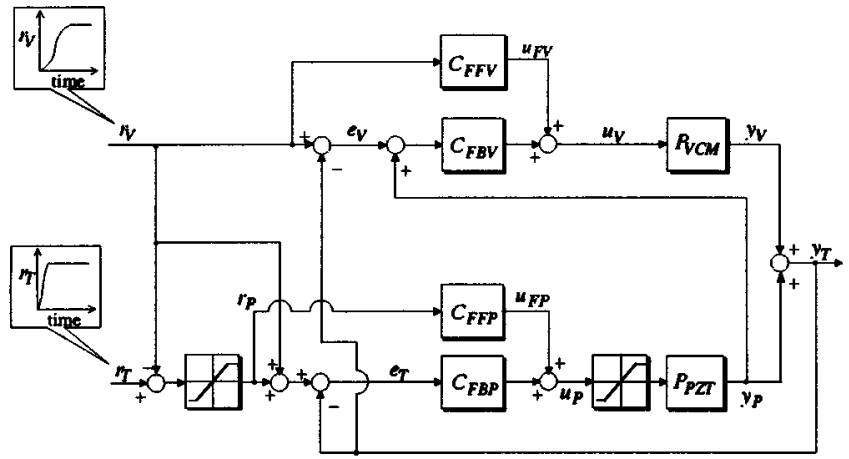

Fig. 12. Short-span seek controller with feedforward saturation.

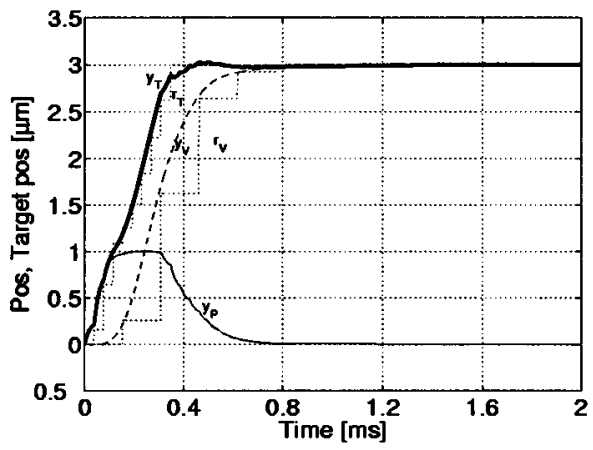

Fig. 13. 3- $\mu \mathrm{m}$ step response without resonance modes (Fig. 12).

head can read or write data on the data track. The response is such that the PZT actuator first moves to the target track. After the head is on track, the PZT actuator then returns to its stroke center to cancel out the movement of the VCM actuator.

In the block diagram of Fig. 10, the relation of the two feedforward compensators is $C_{F F P}=-C_{F F V P}$. When a feedforward trajectory $r_{P}$ for the PZT actuator (relative reference position for the micro-actuator) is defined as

$$
r_{P}=r_{T}-r_{V}
$$

then the feedforward input signal to the PZT actuator is $r_{F P}=$ $C_{F F P} r_{P}$. The meaning of the above equation comes from the relation $y_{T}=y_{V}+y_{P}$. In the experiment, we need to store or calculate four trajectories: the VCM position reference trajectory $r_{V}$, its feedforward input signal $u_{F V}$, the total position reference trajectory $r_{T}$, and the PZT feedforward input signal $u_{F P}$.

\section{B. Short-Span Seeking (Response Over PZT Stroke Limit)}

In this section, we will discuss the short-span seeking response when the head moves over the PZT actuator's stroke limit. We present two structures for the decoupled feedforward control system for the short-span seeking operation. One structure shown in Fig. 12 has the PZT saturation model inserted into the feedforward block for the PZT actuator in order to generate a limited reference feedforward signal $r_{P}$ for the PZT actuator. The difference between Figs. 10 and 12 is only the feedforward saturation block. When the plant does not have resonance modes at high frequency, the responses with this structure for a dual-stage actuator are effective, as shown in Fig. 13. The total head position $y_{T}$ can accurately follow the reference trajectory 


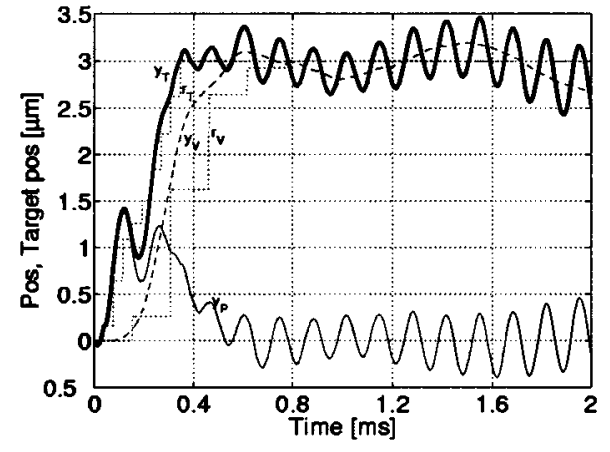

Fig. 14. 3- $\mu \mathrm{m}$ step response with resonance modes (Fig. 12).

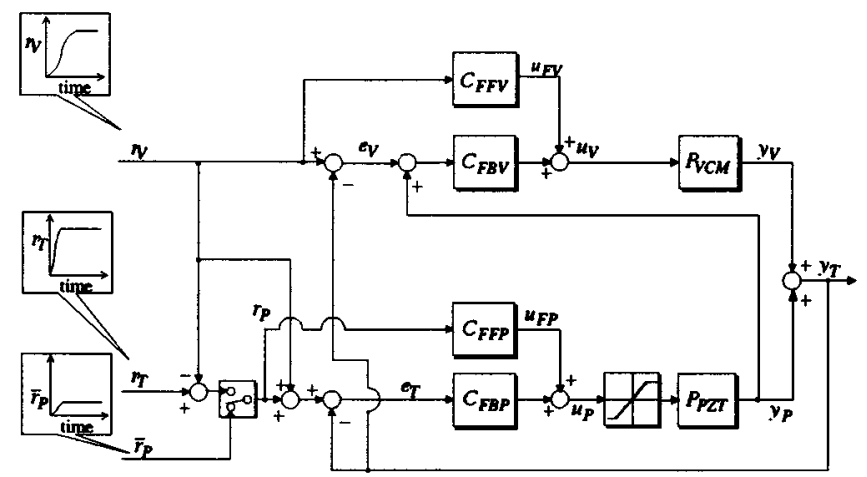

Fig. 15. Short-span seek controller with switch.

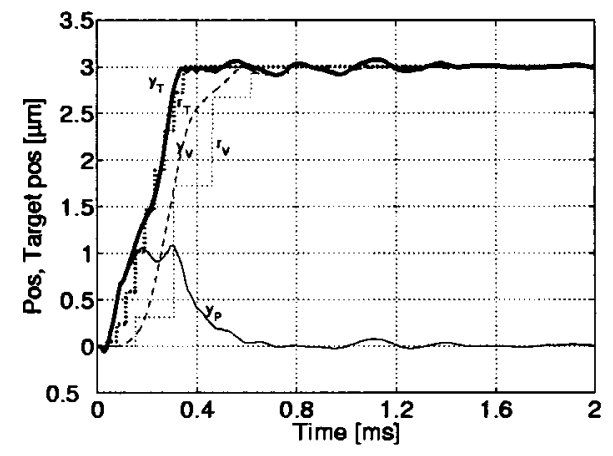

Fig. 16. 3- $\mu \mathrm{m}$ step response with resonance modes (Fig. 15).

$r_{T}$. However, if the plant has resonance modes, the head position response becomes vibratory, as shown in Fig. 14. Since the disadvantage of this method is that we cannot directly specify the PZT actuator trajectory $r_{P}$, the reference trajectory is steep and discontinuous at the beginning acceleration period.

To overcome this disadvantage, we propose a second structure shown in Fig. 15. Instead of the PZT saturation model, we prepare a smooth $1-\mu \mathrm{m}$ reference trajectory $\bar{r}_{P}$ for the PZT actuator and the switch. The sum of the PZT reference trajectory $\bar{r}_{P}$ and the VCM reference trajectory $r_{V}$ is the reference trajectory for the head position $y_{T}$. When the head position reaches the target track, the switch changes its pass loop from the lower one to the upper one so that the PZT actuator moves to cancel out the response of the VCM actuator. Fig. 16 shows the $3-\mu \mathrm{m}$ step response of the dual-stage actuator with resonance modes using the second structure. The PZT actuator works at both the beginning and the final stages of track seeking. It takes about

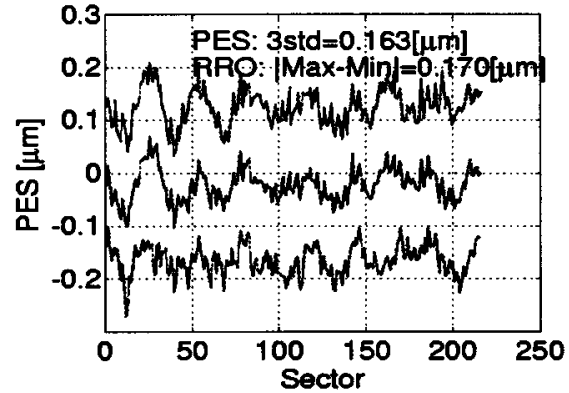

Fig. 17. PES measurements (single VCM servo).



Fig. 18. PES measurements (dual-stage servo).

$0.3 \mathrm{~ms}$ for the head position to reach the target track, while it takes about $0.6 \mathrm{~ms}$ for the single $\mathrm{VCM}$ response.

\section{EXPERIMENTAL RESULTS}

The proposed decoupled seeking controller was applied to a digital control setup for a 3.5 -inch magnetic disk drive. The digital controller was based on software routine implementation in a Digital Signal Processor. The parameters for the experiment were the same as those described in Section II. The PZT gain was $\pm 1.5 \mu \mathrm{m} / \pm 5 \mathrm{~V}$.

\section{A. Track-Following}

We evaluated the position accuracy of the track-following control system. Fig. 17 shows position error signals (PES) of the single-stage system controlled by the VCM actuator. Position error is measured over a period of 1000 revolutions at the center of track (horizontal axis is one disk revolution). Fig. 18 shows the position error signals (PES) of the dual-stage system controlled by the decoupled track-following controller. In both figures, the envelope signal of the PES and the average signal (repeatable position error; RRO) are shown. The Min-Max value of dual-stage RRO is $0.111 \mu \mathrm{m}$, about $34 \%$ less than that of the VCM single actuation $(0.170 \mu \mathrm{m})$. The $3 \sigma$ value of nonrepeatable position error (NRRO) with dual-stage is $0.074 \mu \mathrm{m}$, about $45 \%$ less than that of the VCM single actuation $(0.136 \mu \mathrm{m})$.

\section{B. Track-Seeking}

We first evaluated $0.5-\mu \mathrm{m}$ track-seeking responses, which are within the PZT actuator stroke limit. Fig. 19 shows the single VCM actuator response and Fig. 20 shows the dual-stage actuator response with the proposed one-track decoupled seeking controller (shown in Fig. 10). In each figure, the envelope of the responses and the average of 128 responses are shown. The 


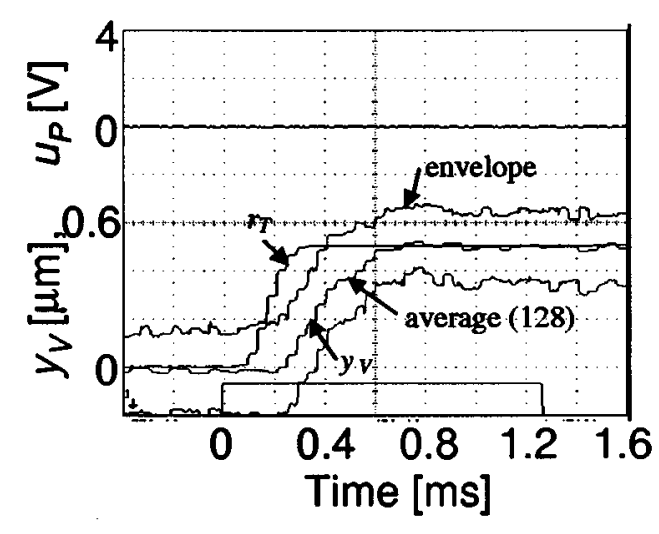

Fig. 19. $\quad 0.5-\mu \mathrm{m}$ seeking response (single VCM servo).



Fig. 20. $\quad 0.5-\mu \mathrm{m}$ seeking response (dual-stage track-seeking servo).

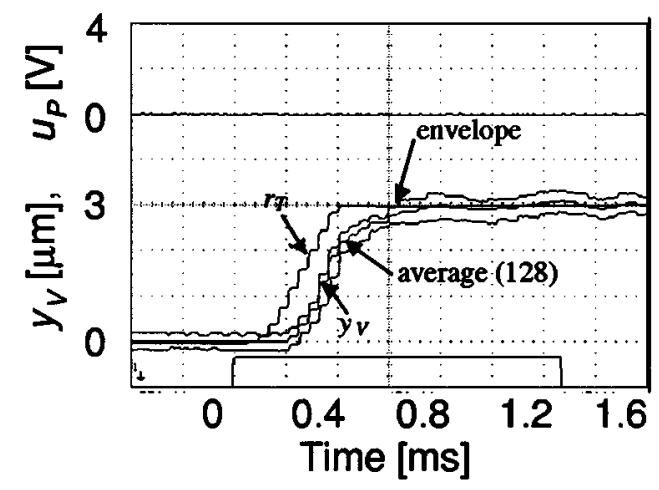

Fig. 21. 3- $\mu \mathrm{m}$ seeking response (single VCM servo).

head position $y_{T}$ of the dual-stage actuator accurately followed the reference signal $r_{T}$. The head reached the target track in $0.3 \mathrm{~ms}$, while the head with the single VCM actuator reached the track in $0.6 \mathrm{~ms}$.

Next, we evaluated 3- $\mu \mathrm{m}$ track-seeking responses, which are over the PZT actuator stroke limit. Fig. 21 shows the single VCM actuator response and Fig. 22 shows the dual-stage actuator response with the proposed short-span decoupled seeking controller (shown in Fig. 15). The head position $y_{T}$ of dual-stage actuator accurately followed the reference signal $r_{T}$. The head

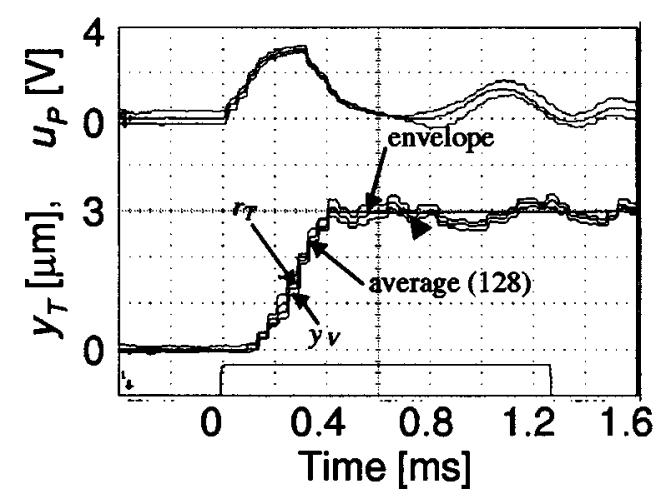

Fig. 22. 3- $\mu \mathrm{m}$ seeking response (dual-stage track-seeking servo).

reached the target track in $0.4 \mathrm{~ms}$, while the head with the single VCM actuator reached the track in $0.7 \mathrm{~ms}$. The input $u_{P}$ for PZT actuator was limited $\pm 3.3 \mathrm{~V}( \pm 1 \mu \mathrm{m})$. The responses were similar to the simulation results and the seeking performance was good.

\section{CONCLUSION}

A decoupled track-seeking controller for a dual-stage actuator was developed. The track-seeking controller was successfully implemented in a magnetic disk drive and effective results were obtained for both simulations and experiments.

\section{ACKNOWLEDGMENT}

The authors would like to thank Y. Li of University of California at Berkeley, Dr. T. Yamaguchi, and I. Naniwa of Hitachi, Ltd. for their valuable help and suggestions.

\section{REFERENCES}

[1] K. Mori et al., "A dual-stage magnetic disk drive actuator using a piezoelectric drive for a high track density," IEEE Trans. Magn., vol. 27, no. 6, pp. 5298-5300, 1991.

[2] I. Naniwa et al., "Low voltage driven piggy-back actuator of hard disk drives," IEEE Int. Micro Electro Mechanical Syst. Conf.: MEMS'99.

[3] R. B. Evans et al., "Piezoelectric microactuator for dual stage control," IEEE Trans. Magn., vol. 35, no. 2, pp. 977-982, 1999.

[4] S. Yoshikazu et al., "Piezoelectric piggy-back microactuator for hard disk drive," IEEE Trans. Magn., vol. 35, no. 2, pp. 983-987, 1999.

[5] D. A. Horsley et al., "Precision positioning using a microfabricated electrostatic actuator," IEEE Trans. Magn., vol. 35, no. 2, pp. 993-999, 1999.

[6] H. Fujita et al., "A microactuator for head positioning system of hard disk drives,” IEEE Trans. Magn., vol. 35, no. 2, pp. 1006-1010, 1999.

[7] T. Semba et al., "Dual-stage servo controller for HDD using MEMS microactuator," IEEE Trans. Magn., vol. 35, no. 5, pp. 2271-2273, 1999.

[8] D. Hernandez et al., "Dual-stage track-following servo design for hard disk drives," in Proc. American Control Conf., 1999, pp. 4116-4121.

[9] S. J. Schroeck and W. C. Messner, "On controller design for linear timeinvariant dual-input single-output systems," in Proc. American Control Conf., 1999, pp. 4122-4126.

[10] M. Kobayashi et al., "Multi-sensing servo with carriage-acceleration feedback for magnetic disk drives," in Proc. American Control Conf., 1998, pp. 3038-3042.

[11] M. Kobayashi et al., "Multirate zero phase error feedforward control for magnetic disk drives" (in Japanese), in JSME Proc. IIP'98, 1998, pp. 21-22. 